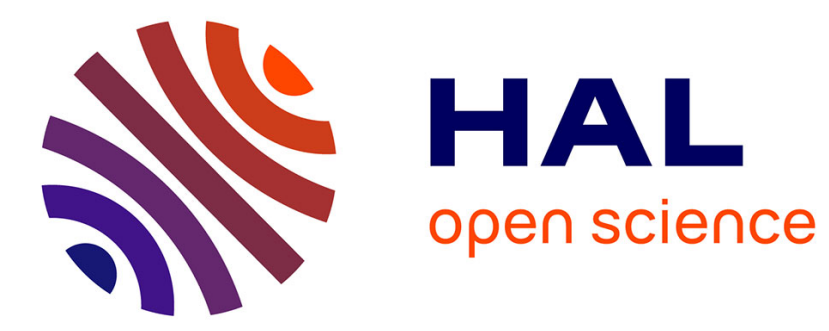

\title{
Blepharospasm, dry eye and extractable nuclear antigen antibodies
}

\author{
B.C. Girard, M. Abdellaoui, G. de Saint Sauveur, A. Huang, P. Lévy
}

\section{To cite this version:}

B.C. Girard, M. Abdellaoui, G. de Saint Sauveur, A. Huang, P. Lévy. Blepharospasm, dry eye and extractable nuclear antigen antibodies. Journal Français d'Ophtalmologie, 2020, 43 (7), pp.e211-e215. 10.1016/j.jfo.2020.06.001 . hal-03201761

\section{HAL Id: hal-03201761 \\ https://hal.sorbonne-universite.fr/hal-03201761}

Submitted on 19 Apr 2021

HAL is a multi-disciplinary open access archive for the deposit and dissemination of scientific research documents, whether they are published or not. The documents may come from teaching and research institutions in France or abroad, or from public or private research centers.
L'archive ouverte pluridisciplinaire HAL, est destinée au dépôt et à la diffusion de documents scientifiques de niveau recherche, publiés ou non, émanant des établissements d'enseignement et de recherche français ou étrangers, des laboratoires publics ou privés. 
Blépharospasme et anticorps anti SSA et anti SSB.

Blépharospasme, sécheresse oculaire et anticorps anti nucléaires solubles.

Titre court

Blépharospasme et anticorps anti SSA et anti SSB. 
Introduction :

Le blépharospasme essentiel (BSE) est une dystonie cérébrale profonde, d'étiologie inconnue. La fréquence de la sécheresse oculaire évoque chez certains patients une possible association avec des maladies auto-immunes tels que le syndrome de Gougerot-Sjögren (SS). L'injection intra musculaire de neurotoxine botulique type A est le seul traitement ayant réellement amélioré cette pathologie ces trentes dernières années. Nous avons voulu, sur une cohorte de 71 patients BS+, étudier l’hypothèse auto-immune qui relierait le BSE et le SS,

\section{Matériel et Méthode}

Nous avons réalisé une étude prospective de juin 2016 à juin 2018, monocentrique, ouverte, concernant des patients venant à la consultation d'ophtalmologie pour dystonie faciale évoquant un blépharospasme pour étudier leur test de Schirmer ainsi que leur profil sérique immunologique en faveur d'un SS. Nous avons recueilli les valeurs des test de Schirmer I à 5 minutes à l'oeil droit et à l'oeil gauche, nous avons ensuite calculé la moyenne des Schirmer I des 2 yeux. Nous avons ensuite réalisé un prélevement sanguin afin de déterminer le profil sérique immunologique de chacun des patients afin de rechercher un profil en faveur d'un SS.

\section{Résultats}

Notre étude a regroupé 71 patients (142 yeux) dont 61 femmes (85,9\%). Le test de Schirmer I de l'œil droit était en moyenne de $3.08 \mathrm{~mm} \pm 4.33 \mathrm{~mm}$. Le test de Schirmer I de l'œil gauche était en moyenne de $3.19 \mathrm{~mm} \pm 5.00 \mathrm{~mm}$. Sur les 61 femmes de l'étude $(61 / 71), 5$ femmes ont des anticorps anti SSA-SSB positifs soit $8,19 \%$ de notre population féminine étudiée (ou 7\% si l'on considère les 71 patients au total).

\section{Conclusion}

Cette étude illustre la possible association entre la présence d'une maladie auto-immune et la survenue un BSE.

\section{Introduction:}

Essential blepharospasm (BSE) is a profound cerebral dystonia of unknown etiology. The frequency of dry eye evokes in some patients a possible association with autoimmune diseases such as Gougerot-Sjögren syndrome (SS). Intramuscular injection of botulinum neurotoxin type A is the only treatment that has really improved this pathology in the last 30 years. We wanted, on a cohort of $71 \mathrm{BS}+$ patients, to study the autoimmune hypothesis that would link the BSE and the SS,

\section{Material and method}

We carried out a prospective study from June 2016 to June 2018, monocentric, open, concerning patients coming to the consultation of ophthalmology for facial dystonia evoking a blepharospasm to study their Schirmer test as well as their serum immunological profile in favor of a SS. We collected the values of the Schirmer I test at 5 minutes in the right eye and the left eye, then we calculated the average of Schirmer I of both eyes. We then performed a blood test to determine the serum immunological profile of each patient to search for a profile in favor of an SS.

\section{Results}

Our study grouped 71 patients (142 eyes) including 61 women (85.9\%). The Schirmer I test of the right eye averaged $3.08 \mathrm{~mm} \pm 4.33 \mathrm{~mm}$. The Schirmer I test of the left eye averaged $3.19 \mathrm{~mm} \pm$ $5.00 \mathrm{~mm}$. Of the 61 women in the study (61/71), 5 women had anti-SSA-SSB antibodies that was $8.19 \%$ of our female population studied (or $7 \%$ if we consider the 71 patients in total).

\section{Conclusion}


This study illustrates the possible association between the presence of an autoimmune disease and the occurrence of a BSE. 
Blépharospasme et anticorps anti SSA-SSB

1 Blépharospasme, sécheresse oculaire et anticorps anti-nucléaires

2 solubles.

3

$4 \quad$ Titre court

5 Blépharospasme et anticorps anti SSA-SSB

6

$7 \quad$ Titre anglais

8 Blepharospasm, dry eye syndrome and ENA antibodies.

9 


\section{Résumé}

\section{Objectif.}

3 Etudier s'il existe un lien entre le blépharospasme essentiel et le syndrome de

4 Sjögren en analysant la présence d'anticorps nucléaires solubles dans cette

5 population,

\section{Méthodes.}

7 Soixante douze patients atteints de blépharospasme essentiel (BSE) ont été

8 inclus dans cette étude. Nous avons éliminé les patients atteints de spasme

9 hémifacial, ou de blépharospasme secondaire à une pathologie cornéenne avérée. Nous avons recueilli les valeurs des test de Schirmer I et les résultats des anticorps anti SSA et anti SSB.

Résultats.

Notre étude a regroupé 72 patients (144 yeux) dont 62 femmes (86.1\%). L’âge moyen était de 74.3 ans \pm 10.73 . Le test de Schirmer I était en moyenne de $3.14 \mathrm{~mm} \pm 4.00 \mathrm{~mm}$. Cinq femmes ( $8 \%$ de cette population féminine) ont révélés des anticorps anti SSA-SSB positifs. Leur moyenne d'âge était de 65.66 ans \pm 13.24 alors que les patients anticorps négatifs avaient une moyenne d'âge de $75.42 \pm 9.27$. Il n'existait pas de différence statistiquement significative entre leur test de Schimer I et celui des patients anticorps négatifs.

\section{Conclusion.}


Blépharospasme et anticorps anti SSA-SSB

1 Cette étude illustre la possible association entre la présence d'une maladie de

2 Sjögren et la présence d'un BSE justifiant la recherche d'anticorps anti SSA et $3 \quad$ SSB chez ces patients.

4

5 Les auteurs déclarent n'avoir aucun conflit d'intérêts en relation avec cet

6 article.

7

8 Mots clefs

9 Blépharospasme; Syndrome de Gougerot-Sjögren; syndrome oculaire sec ;

10 sécheresse oculaire ; Schirmer; anticorps nucléaires solubles; anticorps anti-

11 SSA ; anticorps anti-SSB ; neurotoxine botulique A. 


\section{Summary}

3 Purpose.

4 The goal of this study is to determine a link between benign essential

5 blepharospasm and Sjogren's syndrome by analyzing the presence of

6 extractable nuclear antigens in this population,

7 Methods.

8 Seventy-two patients with benign essential blepharospasm (BEB) were

9 included in this study. We eliminated patients with hemifacial spasm or blepharospasm secondary to corneal pathology. We collected the values of the Schirmer I test and the results of the anti SSA and anti SSB antibodies.

\section{Results}

Our study included 72 patients (144 eyes) whose 62 women (86.1\%). Mean age was 74.3 years \pm 10.73 . Average Schirmer I test was $3.14 \mathrm{~mm} \pm 4.00 \mathrm{~mm}$. Five women ( $8 \%$ of this female population) had positive anti-SSA-SSB antibodies. Their mean age was 65.66 years \pm 13.24 whereas the negative antibody patients had an average age of $75.42 \pm 9.27$. There was no statistically significant difference between their Schimer I test and the Schirmer I of negative antibody population.

\section{Conclusion.}


Blépharospasme et anticorps anti SSA-SSB

1 This study illustrates the possible association between the presence of Sjögren's

2 syndrome and the occurrence of a BEB justifying the search for anti-SSA and

3

4

$5 \quad$ Key word

6 Blepharospasm, Sjögren Syndrome, Dry Eye Syndrome, Dry eye, Schirmer,

7 ENA antibodies, anti- SSA antibodies, anti-SSB antibodies, Botulinum

8 NeuroToxin A.

9 
Blépharospasme et anticorps anti SSA-SSB

2 Le blépharospasme essentiel (BSE) est une dystonie localisée, de fonction,

3 d'origine cérébrale profonde [1,2]. Cette pathologie reste méconnue et sous

4 diagnostiquée. L'injection intramusculaire de neurotoxine botulique A

$5 \quad(\mathrm{NTB} / \mathrm{A})$ est le seul traitement qui ait réellement amélioré cette pathologie

6 depuis une trentaine d'année $[3,4,5]$ et qui a progressivement permis une

7 classification plus précise des dystonies de la face, du syndrome de Meige au

8 blépharospasme [6,7]. L'étiologie du BSE reste obscure. La fréquence de la

9 sécheresse oculaire associée [8] évoque chez certains une possible association avec les maladies auto-immunes et en particulier le syndrome de Sjögren (SS)

[7,9]. Cette hypothèse est très importante à évaluer, le syndrome sec pouvant être l'origine d'une augmentation du clignement et pour certains auteurs l'origine du blépharospasme [10]. Le BSE est actuellement un diagnostic clinique. Les recherches neurologiques et neuroradiologiques s'intéressent à l'IRM fonctionnelle (IRMf) afin de localiser la zone cérébrale déficiente (noyaux gris centraux), mais l'IRMf reste difficile à mettre en œuvre dans le BSE car le mouvement n'est pas volontaire $[11,12]$. La présence d'une sécheresse oculaire dans 60 à 80\% des patients atteints de BSE [8], est importante à diagnostiquer et à traiter dans les deux formes de BS, essentiel ou secondaire. Cependant le traitement de fond de la maladie est très différent selon qu'il s'agit d'un blépharospasme essentiel ou d'un blépharospasme secondaire à une maladie auto-immune. Nous avons voulu approfondir cette 
Blépharospasme et anticorps anti SSA-SSB

1 hypothèse auto-immune qui relierait le blépharospasme (BS) et le syndrome de 2 Gougerot-Sjögren.

3 


\section{Matériel et méthodes}

2 Nous avons réalisé une étude prospective de juin 2016 à juin 2018,

3 monocentrique, ouverte, concernant les patients venant à la consultation

4 d'ophtalmologie, pour dystonie faciale évoquant un blépharospasme afin

5 d'analyser leur sécheresse oculaire et leur profil sérique immunologique en

6 faveur d'un syndrome de Gougerot-Sjögren. Les patients venaient de toute la

7 France, adressés par des ophtalmologistes, des neurologues, ou par

8 l'association de malades (AMADYS) vers un centre hospitalier de traitement

9 des dystonies faciales (blépharospasme). Nous avons éliminé de l'étude les patients atteints de spasme hémi-facial et les patients adressés pour clignement anormal lié à une pathologie de surface oculaire (blépharospasme secondaire) pour ne garder que les patients atteints de blépharospasme essentiel, dystonie orbiculaire bilatérale. Nous avons recueilli les valeurs des tests de Schirmer I à 5 minutes, sans anesthésie, de l'œil droit et de l'œil gauche. Pour l'étude nous avons fait la moyenne des Schirmer I des 2 yeux. Nous avons prélevé un échantillon sanguin chez tous ces patients, après avoir obtenu leur consentement éclairé, afin de rechercher la présence d'anticorps anti nucléaires solubles, avec profil immunologique en faveur d'une étiologie systémique (Syndrome de Gougerot-Sjögren). Les prélèvements sanguins étaient faits soit en consultation hospitalière d'ophtalmologie sur place, le jour de l'examen, soit en laboratoire extérieur à l'hôpital. Les résultats 
1 immunologiques à la recherche d'anticorps antinucléaires solubles provenaient

2 de différents laboratoires. La recherche a été effectuée de manière simultanée

3 pour les anticorps anti-nucléaires solubles anti-SSA, anti-SSB, anti-Sm, anti-

4 RNP et pour les auto-anticorps anti Ro60/SSA, anti Ro52/SSA [5], anti

5 La/SSB, Sm-RNP, Scl-70, Jo1, et leur aspect en fluorescence. Le résultat était 6 considéré selon les normes du laboratoire.

7

$8 \quad$ Statistiques :

9 Les variables quantitatives ont été analysées avec le test de Mann Whitney, le 10 test des rangs de Wilcoxon et le test de Spearman. Le seuil de significativité

11 était de 0.05. Le logiciel utilisé était Statview (V5.0, SAS insitute). 
Blépharospasme et anticorps anti SSA-SSB

\section{Résultats}

2 Notre étude a regroupé 72 patients atteints de BS (144 yeux) dont 62 femmes

3 (86.1\%). La moyenne d'âge de la cohorte est de 74.3 ans, \pm 10.73 , avec une

4 médiane à 75 ans (range : 37-93). La moyenne d'âge des hommes était de 73.2

5 ans $\pm 15,06$, avec une médiane à 74 ans (range 37-87). La moyenne d'âge des

6 femmes était de 74.48 ans \pm 10.01 , avec une médiane à 76 ans (range 45-93)

7 (Tableau 1). Il n'existe pas de différence statistiquement significative d'âge

8 entre les hommes et les femmes $(\mathrm{p}<0,01)$.

9 Tous les patients avaient un blépharospasme (BS) et étaient traités par des injections trimestrielles de NTB/A A dans les muscles orbiculaires et péri orbiculaires. Le délai de diagnostic (temps entre le début de la maladie et la consultation donnant le diagnostic) pour les 38 patients pour lesquels cette donnée était disponible était de 37.92 mois en moyenne, écart-type \pm 71.35 (range de 2 à 408 mois, soit de 2 mois à 34 ans !). Les patients ont consulté 3 médecins en moyenne \pm 6 (range 1 à 8 médecins) avant d'avoir connaissance de leur maladie.

Le test de Schirmer I était réalisé systématiquement à l'arrivée des patients, qui n’avaient pas instillé de collyres depuis la veille au soir (Tableau 2).

Le test de Schirmer I de l'œil droit était en moyenne de $3.08 \mathrm{~mm} \pm 4.33 \mathrm{~mm}$.

Le test de Schirmer I de l'œil gauche était en moyenne de $3.19 \mathrm{~mm} \pm 5.00 \mathrm{~mm}$. 
1 Il n'existe pas de différence statistiquement significative entre les tests de

2 Schirmer droits et gauches $(\mathrm{p}<0,05)$ et pour l'étude nous avons considéré la

3 moyenne de test de Schirmer entre les deux yeux. Les tests de Schirmer I

4 (moyenne $\mathrm{OD}$ et $\mathrm{OG}$ ) était de $3.14 \mathrm{~mm} \pm 4$ en moyenne arithmétique sur cette

5 cohorte de 72 patients atteints de BS.

6 Chez les hommes les tests de Schirmer était de $1.1 \mathrm{~mm} \pm 0.87$ et chez les

7 femmes de $3.47 \mathrm{~mm} \pm 4.21$. L'analyse des tests de Schirmer en fonction du sexe montrait une différence statistiquement significative $(\mathrm{p}=0.0138)$ avec un test de Schirmer plus faible chez les hommes. Par contre il n'y avait pas de lien entre l'âge, le sexe et le Schirmer $(\mathrm{p}=0.3457)$, le Schirmer étant inférieur à 5 mm chez $82 \%$ des patients, âge et sexe confondus (Tableau 3).

Sur les 62 femmes de l'étude (62/72), 5 femmes ont des anticorps anti SSASSB positifs (Tableau 4) soit $8 \%$ de notre population féminine étudiée (ou 7\% si l'on considère les 72 patients au total).

La moyenne des tests de Schirmer I des cinq patientes BS avec anticorps SS positifs est de $3.66 \mathrm{~mm} \pm 5.09$, la moyenne des 67 patients (hommes et femmes) BS avec anticorps SS négatifs est $\mathbf{3 . 0 5} \mathrm{mm} \pm 3.9$.

Nous avons analysé s'il existait un lien entre sexe et résultats positifs des anticorps antiSSA et SSB. Nous avons établi un groupe d'hommes BS qui s'avèrent être tous anticorps solubles négatifs, un deuxième groupe des femmes BS anticorps négatifs et un troisième groupe de femmes BS anticorps 
Blépharospasme et anticorps anti SSA-SSB

1 positifs. Il apparait une différence statistiquement significative $(\mathrm{p}<0.05)$ entre

2 ces groupes concernant l'âge d'apparition du BS (Tableau 3). Les femmes BS

3 possèdant des anticorps solubles positifs étaient plus jeunes de 10 ans que

4 celles ayant un BS sans anticorps.

5

6

7 
Blépharospasme et anticorps anti SSA-SSB

\section{Discussion et conclusion}

2 Le syndrome oculaire sec en France est fréquent et atteint $30 \%$ de la population

3 féminine de plus de 65 ans [13]. Il peut être primitif ou associé à d'autres

4 pathologies. Le SS est une des étiologies du syndrome oculaire sec [14,15]. La

5 prévalence du SS primitif dans le monde varie de $0,3 \%$ à $2,7 \%$ selon les études

$6 \quad[16,17]$ et est d'environ de 0,5 à $1 \%$ en France avec une incidence variant selon

7 les études de 4 à 5 pour 100000 [18]. Dans la population uniquement féminine la prévalence SS est de fait identique, de 0,2 à $1 \%$ [19]. En effet la maladie de Sjögren dans sa forme primitive touche essentiellement les femmes $(9: 1)$ [20,21] dont l'âge moyen d'apparition se situe vers 50 ans [13]. Même si la sérologie est non spécifique du SS [13,22] nous sommes interpelés par la fréquence des anticorps antinucléaires solubles retrouvés à $8 \%$ dans notre étude, alors que le blépharospasme est une maladie peu fréquente. $\mathrm{La}$ prévalence du blépharospasme est mal connue, avec une incidence de 3.2/100 000 en Sardaigne [23] à 3.6/100 000 en Europe [24]. Le BSE touche plus fréquemment les femmes avec un sex ratio de 2/3. Le BSE survient vers 60 ans $[7,25]$ donc dix ans plus tardivement que le SS $[13,21]$. Cependant si nous étudions nos groupes de patients, les femmes BS ayant une sérologie positive anti SSA-SSB ont 65 ans \pm 13 et sont plus jeunes que les femmes BS anticorps négatifs qui ont 75 ans \pm 9 valeurs conformes aux données de la littérature. La valeur du test de Schirmer I n'est pas statistiquement différente entre ces deux 
1 groupes (Tableau 2). La valeur normale d'un test de Schirmer est supérieure à

$215 \mathrm{~mm}$ à 5 minutes. En dessous de $3 \mathrm{~mm}$, on parle d'un syndrome sec oculaire

3 sévère [26]. Les patients atteints de BSE ont un syndrome oculaire sec sévère

4 dans 60 à $80 \%$ des cas [8], ce que nous retrouvons dans cette étude où $82 \%$

5 des BSE ont un Schirmer inférieur à $5 \mathrm{~mm}$. Nous sommes conscient de la

6 faiblesse de notre étude sur l'œil sec, en nous limitant à l'analyse du Schirmer

7 I, sans utiliser l'OSDI, le BUT, l'aspect à la lampe à fente, et les nouvelles

8 techniques d'exploration des larmes, pour des raisons financières et

9 temporelles. D'autres études doivent venir confirmer ces données. Pour

Jankovic, pionnier dans le diagnostic et le traitement du BSE, il n'est pas rare de trouver le BSE associé à une maladie auto-immune. Il rapporte un cas de BSE associé à un SS [7,9] et insiste oralement sur cette éventualité lors de congrès récents. Les résultats de notre étude révèlent une fréquence huit à vingt-six fois supérieure d'anticorps anti SSA-SSB, par rapport à la fréquence attendue dans une population féminine standard normale $(0.3$ à $1 \%)$ $[16,17,19]$. Cette découverte confirme donc les intuitions de Jankovic et ouvre une nouvelle question concernant la relation entre syndrome oculaire sévère, syndrome de Sjögren et BSE. A l'inverse, seulement 1/3 des syndromes oculaires secs suivis sur onze ans, ont évolué vers un syndrome de GougerotSjögren [26] et aucun n'a évolué vers un BSE. Le syndrome oculaire sec n'est pas à l'origine du SS, ni du BSE. L'origine du BSE n'est pas dans la gravité du syndrome sec oculaire mais neurologique, même si les premiers signes 
1 cliniques se confondent avec une insuffisance lacrymale. Le syndrome oculaire

2 sec observé dans le BSE est cliniquement plus sévère qu'un syndrome oculaire

3 sec isolé, invalidant, associant brulures oculaires, picotement des yeux et des

4 bords des paupières, ne cessant pas la nuit, avec une photophobie intense,

5 s'accompagnant d'une contracture involontaire des muscles corrugators,

6 depressor supercilii, procerus, et orbiculaires orbitaires, pré-septal et pré-tarsal,

7 et reste particulier avec un profil immunologique différent des syndromes

8 oculaires secs isolés [28]. Le profil immunologique des larmes et de la

9 conjonctive montre un niveau d'inflammation augmenté ( IL1, IL6, IL8, TNF alfa), avec un processus inflammatoire chronique qui aggrave le processus inflammatoire du syndrome oculaire sec qui auto alimente la sécheresse oculaire [26]. La réduction du battement des paupières le long de la surface oculaire par les injections régulières de NTB/A améliore l'OSDI [28]. Les injections trimestrielles de NTB/A dans les muscles orbiculaires améliorent les larmes dans le SS en quantité et en qualité [29] malgré une action inhibitrice de la NTB/A si elle était injectée dans la glande lacrymale principale [30]. Peut-on suspecter le SS lors d'un examen d'un patient atteint de BS ? Le syndrome oculaire sec du BS, cliniquement ou sur le Schirmer n'est pas différent, qu'il s'agisse de patientes SS ou de patientes ayant un BSE. Dans notre étude les 5 femmes atteintes de blépharospasme (BS) et ayant des anticorps positifs au Sjögren (SSA ou SSB) ont un test de Schirmer I légèrement supérieur bien que non statistiquement significatif, à celles qui ont un BSE isolé. Cette donnée 
1 pourrait-elle être en faveur d'un syndrome oculaire sec moins sévère dans le

2 blépharospasme secondaire observé chez les patients ayant un éventiuel SS

3 (BSS) que dans le BSE ? Cependant cette donnée n'est pas statistiquement

$4 \quad$ valable et cliniquement le spasme orbiculaire était pour ces deux populations

5 de patientes identiques. Il ne s'agissait pas de clignement accéléré d'une

6 kératite sèche comme il est fréquent de l'observer dans le SS. La dystonie

7 contractait les muscles orbiculaires, mais aussi les corrugators, les procerus et

8 les dépressors supercilii. Il n'y avait aucun signe dans l'attitude des patients laissant entrevoir qu'il pouvait s'agir de blépharospasme secondaire.

Leurs clignements aberrants et dystoniques n'étaient pas modifiable par la surprise , l'ordre ou la volonté. Non distractibles, ils était aussi prégnants que dans les blépharospasmes habituels. Ces patients sont-ils atteints d'un BSE, associé ou non à un SS à anticorps positifs, ou s'agit-il d'un SS avec forte participation des contractions musculaires (blépharospasme secondaire)?

S'agit-il des deux maladies associées ou d'une seule pathologie ? Pour les cliniciens, il reste essentiel de faire la différence entre BSE et BSS afin d'orienter ces patients en Médecine interne, dans les centres spécialisés en maladies systémiques afin de leur faire bénéficier du bilan (recherche des maladies systémiques, dosage des anticorps anti ADN natif, anti-RNP, etc..., biopsies des glandes salivaires accessoires, sécheresse des autres muqueuses, complications respiratoires, cardiaques et articulaires) et des thérapeutiques spécifiques (immunomodulateurs systémiques), tout en traitant 
1 le syndrome oculaire sec et le blépharospasme (injections de NTB/A dans les

2 muscles orbiculaires et péri-orbiculaires). Cette étude a permis de constater

3 la fréquence de positivité aux anticorps anti SSA ou SSB, et nous suggérons la

4 nécessité d'améliorer la prise en charge des BS par la recherche sérologique

5 des anticorps nucléaires solubles à la première consultation de blépharospasme

6 afin de distinguer le BSE d'un BS, essentiel ou secondaire, associé au

7 syndrome de Sjögren, d'autant qu'il s'agit de femmes, plus jeunes que l'âge

8 habituel de survenue du BSE. D'autres études devront valider cette hypothèse

9 et approfondir l'existance d'un syndrome de Sjögren associé à une

blépharospasme. 
Blépharospasme et anticorps anti SSA-SSB

2 Références

3 [1] Henderson JW. Essential blepharospasm. Trans Am Ophthalmol Soc 1956;

$4 \quad 54 ; 453-520$.

5 [2] Jankovic J, Nutt JG : Blepharospasm and cranial-cervical dystonia (Meige's

6 syndrome) familial occurrence in facial dyskinesias. Jankovic J, Tolosa E.,

7 Edts Raven press, New York, Adv Neurol 1988, 49, 117-23.

8 [3] Engstrom PF, Arnoult JB, Mazow ML, Prager TC, Wilkins RB, Byrd WA,

9 Hofmann RJ. Effectiveness of botulinum toxin therapy for essential blepharospasm. Ophthalmology 1987 94:971-5.

[4] Patel B, Anderson RL. Diagnosis and management of essential

blepharospasm. Ophthalmic Pract 1993; 11: 293-300.

[5]Skarf JB, Scharpe JA. Essential blepharospasm : a review of medical management and a therapeutic trial of choline chloride. Am J Ophthalmol 1981;92:742.

[6] Meige H. Les convulsions de la face : une forme clinique de la convulsion faciale, bilatérale et médiane. Rev Neurol (Paris) 1910 ; 10 :437-43. and pathological findings in 100 patients. Ann Neurol. 1983; 13:402-11. 
Blépharospasme et anticorps anti SSA-SSB

1 [8] Girard B, Levy P. Dry-eye syndrome in Benign Essential Blepharospasm. J

2 Fr Ophtalm, 2019 “doi : 10.1016/j.jfo.2019.06.007”

3 [9] Jankovic J, Patten BM. Blepharospasm and autoimmune diseases. Mov

4 Disord 1987;2,3, 159-163.

5 [10] Evinger C, Bao JB, Powers AS, Kassem IS, Schicatano EJ, Henriquez

6 VM, et al. Dry eye, blinking, and blepharospasm. Mov Disord 2002;17 Suppl $7 \quad 2: S 75-8$.

8 [11] Schmidt KE, Linden DE, Goebel R, Zanella FE, Lanfermann H and 9 Zubcov AA. Striatal activation during blepharospasm revealed by fMRI.

Neurology 2003; 60 11: 1738-43.

[12] Dimitrova A, Weber J, Maschke M, Elles HG, Kolb FP, Forsting M, et al. Eyeblink-related areas in human cerebellum as shown by fMRI. Hum Brain Mapp 2002; 17 2: 100-15.

[13] Aikaterini Liapi A, Horisberger F-S, Spertini F, Ribi C. Syndrome de Sjögren : quand le suspecter et comment le confirmer ? Rev Med Suisse $2016 ; 12: 698-702$.

[14] Vitali C. Classification criteria for Sjögren's syndrome: a revised version of the European criteria proposed by the American-European consensus group. Ann Rheum Dis 2003;62:94-5.

$2003 ; 1: 22928-33$. 
Blépharospasme et anticorps anti SSA-SSB

1 [15] Both T, Dalm V, van Hagen PM, van Daele PL. Reviewing primary

2 Sjögren syndrome : beyound the dryness. From pathophysiology to diagnosis

3 and treatment. Int J Med Sci 2017;14:191-200.

4 [16] Miyasaka N. Epidemiology and pathogenesis of Sjögren’s syndrome.

5 Nihon Rinsho Jpn J Clin Med 1995;53:2367-70. (tableau genial)

6 [17] Jacobsson LT, Axell TE, Hansen BU, Henricsson VJ, Larsson A,

7 Lieberkind K, et al. Dry eyes or mouth - an epidemiological study in Swedish

8 adults, with special reference to primary Sjögren's syndrome. J Autoimmun

$9 \quad 1989 ; 2: 521-7$.

10 [18] Binard A, Fautrel B, Jousse-Joulin S, Devauchelle-Pensec V, Saraux A.

11 Incidence et prévalence du syndrome de Gougerot-Sjögren primitif : une analyse systématique de la littérature. J Rhum 2006 ;10-11 :1227.

[19] Dafni UG, Tzioufas AG, Staikos P, Skopouli FN, Moutsopoulos HM. Prevalence of Sjögren'syndrome in a closed rural community. Ann Rheum Dis $1997 ; 56: 521-5$

[20] Patel R, Shahane A. The epidemiology of Sjögren's syndrome. Clin Epidemiol 2014;6:247-55.

[21] Oppliger C, Chizzolini C. Du syndrome sec au syndrome de Sjögren. Rev med Suisse 2003;1:22928-33. 
1 [22] Menéndez A, Gomez-Arbesu J, Escanlar E, Caminal L, Mozo L. Clinical

2 associations of anti-SSA/Ro60 and anti-Ro52/TRIM21 antibodies: Diagnostic

3 utility of their separate detection. Autoimmunity 2013;46:32-9.

4 [23]Cossu G, Mereu A, Deriu M, Melis M, Molari A, Melis G, et al.

5 Prevalence of primary blepharospasm in Sardinia, Italy: a service-based

6 survey. Mov Disord 2006;21:2005-8.

7 [24] The Epidemiological Study of Dystonia in Europe (ESDE) Collaborative

8 Group. A prevalence study of primary dystonia in eight European countries. J

$9 \quad$ Neurol 2000;247:787-92.

[25] Grandas F, Elston J, Quinn N, Marsden CD. Blepharospasm: a review of 264 patients. J Neurol Neurosurg Psychiatry 1988;51:767-72.

[26] Baudouin C, Aragona P, Van Setten G, Rolando M, Irkec M, Benitez del Castillo J, et al. Diagnosing the severity of dry eye : a clear and practical algorithm. Br J Ophthalmol 2014;98:1168-76.

[27] Pertovaara M, Korpela M, Ususitalo H, Pukander J, Miettinen A, Helin H, et al. Clinical follow up study of 87 patients with sicca symptoms (dryness of eyes or mouth or both). Ann Rheum Dis 1999;58:423-7.

[28] Lu R, Huang R, Li K, Zhang X, Yang H, Quan Y, et al. The influence of benign essential blepharospasm on dry eye and ocular inflammation. Am J Ohthalmol 2014;591-7. 
Blépharospasme et anticorps anti SSA-SSB

1 [29] Spiera H, Asbell PA, SimpsonnDM. Botulinum Toxin Increases Tearing

2 in Patients with Sjögren's syndrome : A preliminary Report. J Rheumatol

$3 \quad 1997 ; 24: 1842-3$.

4 [30] Girard B, Piaton JM, Keller P, Nguyen TH. Botulinum neurotoxin A

5 injection for the treatment of epiphora with patent lacrymal ducts. J Fr

6 Ophtalmol 2018;41:343-9.

7

8

9

10 
Blépharospasme et anticorps anti SSA-SSB

\section{$1 \quad$ Legendes des Tableaux}

2 Tableau 1 : caractéristiques démographiques des patients (sexe, âge), et test de

3 Schirmer I.

4 Tableau 2 : Schirmer I selon le sexe, l'âge, et les anticorps anti nucléaires

5 solubles ( $\mathrm{SS}+$ ).

6 Tableau 3 : Syndrome sec chez $82 \%$ des patients.

7 Tableau 4 : profil sérique des patients anticorps SSA-SSB positifs. 
Blépharospasme et anticorps anti SSA et anti SSB.

Tableau 1

\begin{tabular}{|l|l|l|l|l|l|l|l|}
\hline $\begin{array}{l}\text { BS (72 } \\
\text { patients) }\end{array}$ & nombre & $\begin{array}{l}\text { Age } \\
\text { (ans) }\end{array}$ & $\begin{array}{l}\text { Dev. Std } \\
\text { âge }\end{array}$ & $\begin{array}{l}\text { Médiane } \\
\text { âge }\end{array}$ & $\begin{array}{l}\text { Range } \\
\text { ans }\end{array}$ & $\begin{array}{l}\text { Schirmer I } \\
(\mathrm{mm})\end{array}$ & $\begin{array}{l}\text { Dev. Std } \\
\text { Schirmer }\end{array}$ \\
\hline BS & 72 & 74,3 & $\pm 10,73$ & 75 & $37-93$ & 3,14 & $\pm 4,00$ \\
\hline Hommes & 10 & 73,2 & $\pm 15,06$ & 74 & $37-87$ & 1,1 & $\pm 0,87$ \\
\hline Femmes & 62 & 74,48 & $\pm 10,01$ & 76 & $45-93$ & 3,47 & $\pm 4,21$ \\
\hline
\end{tabular}


Blépharospasme et anticorps anti SSA et anti SSB.

Tableau 2

\begin{tabular}{|l|l|l|l|l|l|}
\hline $\begin{array}{l}\text { BS (72 } \\
\text { patients) }\end{array}$ & nombre & $\begin{array}{l}\text { Age } \\
\text { (ans) }\end{array}$ & $\begin{array}{l}\text { Ecart type } \\
\text { âge }\end{array}$ & $\begin{array}{l}\text { Schirmer I } \\
(\mathrm{mm})\end{array}$ & $\begin{array}{l}\text { Ecart type } \\
\text { Schirmer }\end{array}$ \\
\hline & & & & & \\
\hline Hommes SS- & 10 & 73,2 & $\pm 15,06$ & 1,1 & $\pm 0,87$ \\
\hline Femmes SS- & 57 & 75,42 & $\pm 9,27$ & 3,45 & $\pm 4,16$ \\
\hline Femmes SS+ & 5 & 65,66 & $\pm 13,24$ & 3,66 & $\pm 5,09$ \\
\hline
\end{tabular}


Tableau 3

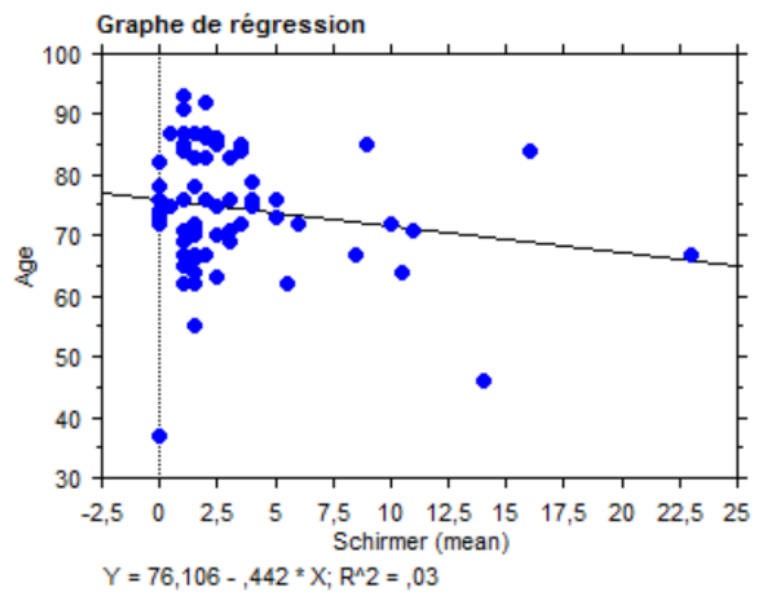

Corrélation de Spearman pourAge, Schirmer (mean)

Somme des carrés des écarts 65802,000

Rho

Valeur de $z$

Valeur de $p$

Rho corrigé pour ex-aequo

$z$ corrigé pour ex-aequo

$p$ corrigé pour ex-aequo

\# ex-aequo, Age

\# ex-aequo, Schirmer (mean)

\begin{tabular}{|r|}
\hline,- 103 \\
\hline,- 864 \\
\hline, 3873 \\
\hline,- 113 \\
\hline,- 943 \\
\hline, 3457 \\
\hline 16 \\
\hline 10 \\
\hline
\end{tabular}

Cas omis (manquants). 
Blépharospasme et anticorps anti SSA et anti SSB.

Tableau 4

\begin{tabular}{|c|c|l|l|}
\hline patientes & $\begin{array}{c}\text { Titre } \\
\text { AAN }\end{array}$ & Aspect en immuno-fluo & Ac antinucléaires solubles \\
\hline 1 & + & Fluorescence mouchetée & Ac anti SSB \\
\hline 2 & + & Fluorescence mouchetée & Ac anti SSA/Ro 52 \\
\hline 3 & + & Fluorescence homogène & Ac anti SSA \\
\hline 4 & + & Fluorescence homogène & Ac anti SSA/Ro 60 \\
\hline 5 & + & Fluorescence homogène & Ac anti SSA/Ro 60 \\
\hline
\end{tabular}

